\title{
Homotopy perturbation method for Ozone decomposition of the second order in aqueous solutions
}

\author{
Z. AYATI, J. BIAZAR, M. PARTOVI
}

\begin{abstract}
In this article the problem of mass transfer of ozone of the second order from a gaseous phase into an aqueous phase has been studied. Homotopy perturbation method is employed to derive an analytical approximation to the solutions of the system of differential equations governing on the problem. Some parametric studies have been included. The effects of the temperature and hydroxyl ion reaction order to the solutions are illustrated by some plots.
\end{abstract}

Additional Key Words and Phrases: Homotopy perturbation method; System of nonlinear differential equation; Ozone decomposition

\section{INTRODUCTION}

The use of ozone has been shown to be efficient in inactivating pathogens and reducing bacterial gill disease in freshwater re-circulation systems. Even in marine systems, for handling environmental contaminants, the use of ozone, is increasingly becoming very popular. Furthermore, ozone has been shown to improve water quality by oxidizing nitrite, natural organic matter (NOM), ammonia, and removal of fine suspended particles as well as re-oxygenation of the water [1-2].

The ozone demand and decomposition behaviour in aqueous solutions need to be quantified. In this study, the ozone decomposition kinetics is being investigated. Biazar [3] used the Adomian decomposition method (ADM) to solve this problem. This paper is to extend the homotopy perturbation method ( HPM ) proposed by He [4-13] to solve the Ozone decomposition. The results of the HPM are compared with those obtained by the ADM for different values of constants. The HPM is useful to obtain exact and approximate solutions of linear and nonlinear differential equations. This reliable analytic approximation to the solution is of great interest. The main reason of this interest is that this analytic approximation 
can be used in many engineering purposes, having this analytic approximation one can easily study the effects of different

parameters to the solution. Mathematical modelling of the problem lead to the following system of two nonlinear differential equations:

$$
\left\{\begin{array}{l}
\frac{d C(t)}{d t}=-K_{D} C(t)^{2}-K_{R} D(t) C(t), \\
\frac{d D(t)}{d t}=-K_{R} D(t) C(t),
\end{array}\right.
$$

where $D(t)$, concentration of NOM fraction with fast ozone demand $(\mathrm{mg} / \mathrm{l})$; $C(t)$, first-order dissolved oxygen concentration at time $t(\mathrm{mg} / \mathrm{l})$; second-order rate $K_{D}$ ozone decomposition rate constant $(1 / \mathrm{min}) . K_{R}$ Constant (1/ mg min). A substantial amount of numerical and analytical work has been invested on this model.

\section{BASIC IDEA OF HOMOTOPY PERTURBATION METHOD}

To illustrate the basic ideas of homotopy perturbation method for solving nonlinear differential equations, we consider the following nonlinear differential equation:

$$
A(u)=f(r), \quad r \in \Omega,
$$

with the following boundary conditions:

$$
B\left(u, \frac{\partial u}{\partial n}\right)=0, \quad r \in \Gamma,
$$

where $\mathrm{A}$ is a general functional operator, $B$ is a boundary operator, $f(r)$ is a known analytical function, and $\Gamma$ is the boundary of the domain $\Omega$ and $\partial / \partial n$ denotes differentiation along the normal drawn outwards from $\Omega$. Generally speaking the operator A can be divided into two parts $L$ and $N$, where $L$ is a linear and $N$ is a nonlinear operator. Eq. (2), therefore, can be rewritten as the following:

$$
L(u)+N(u)-f(r)=0 .
$$


we construct a homotopy of Eq. (2) $v(r, p): \Omega \times[0,1] \rightarrow \square$, which satisfies

$$
H(v, p)=(1-p)\left[L(v)-L\left(u_{0}\right)\right]+p[A(v)-f(r)]=0, \quad p \in[0,1], \quad r \in \Omega .
$$

Which is equivalent to

$$
H(v, p)=L(v)-L\left(u_{0}\right)+p L\left(u_{0}\right)+p[N(v)-f(r)]=0,
$$

Where $p \in[0,1]$, is an embedding parameter, $u_{0}$ is an initial approximation for the solution of Eq. (2), which satisfies the boundary conditions. Obviously, from Eqs. (5) and (6) we will have

$$
H(v, 0)=L(v)-L\left(u_{0}\right)=0, H(v, 1)=A(v)-f(r)=0 .
$$

thus, the changing process of $p$ from zero to unity is just that of $v(r, p)$ from $u_{0}$ to $u_{r}$. In topology, these are called deformation, and $L(v)-L\left(u_{0}\right), A(v)-f(r)$ are called homotopic. Here the embedding parameter is introduced much more naturally, unaffected by artificial factors; further it can be considered as small parameter for $0 \leq p \leq 1$. So it is very natural to assumed that the solution of (5) and (6) can be expressed as a power series in $p$ :

$$
v=v_{0}+p v_{1}+p^{2} v_{2}+\cdots
$$

The approximate solution of Eq. (1), therefore, can be readily obtained:

$$
u=\lim _{p \rightarrow 1} v=v_{0}+v_{1}+v_{2}+\cdots
$$

\section{SOLUTIONS OF THE SYSTEM (1) BY HPM.}

In this section, we will apply the HPM to nonlinear system of equations (1) as follows: 


$$
\left\{\begin{array}{l}
(1-p)\left(\frac{d C}{d t}-\frac{d u_{0}}{d t}\right)+p\left(\frac{d C}{d t}+K_{D} C^{2}+K_{R} C D\right)=0 \\
(1-p)\left(\frac{d D}{d t}-\frac{d v_{0}}{d t}\right)+p\left(\frac{d D}{d t}+K_{R} D C\right)=0
\end{array}\right.
$$

or

$$
\left\{\begin{array}{l}
\frac{d C}{d t}-\frac{d u_{0}}{d t}+p\left(\frac{d u_{0}}{d t}+K_{D} C^{2}+K_{R} C D\right)=0 \\
\frac{d D}{d t}-\frac{d v_{0}}{d t}+p\left(\frac{d v_{0}}{d t}+K_{R} D C\right)=0 .
\end{array}\right.
$$

And the initial approximations are as follows:

$$
\begin{aligned}
& C_{0}=C(0), \\
& D_{0}=D(0),
\end{aligned}
$$

As usual in HPM, consider the solution of Eq (2) is two series:

$$
\begin{aligned}
& C=C_{0}+p C_{1}+p^{2} C_{2}+p^{3} C_{3}+\cdots \\
& D=D_{0}+p D_{1}+p^{2} D_{2}+p^{3} D_{3} \cdots
\end{aligned}
$$

Substituting (13) into (11), and equating the coefficients of the terms with the identical powers of $p$, leads to:

$$
\begin{aligned}
& p^{0}:\left\{\begin{array}{l}
C_{0}=u_{0}=C(0), \\
D_{0}=v_{0}=D(0),
\end{array}\right. \\
& p^{1}:\left\{\begin{array}{l}
C_{1}=-C(0)\left(K_{D} C(0)+K_{R} D(0)\right) t, \\
D_{1}=-K_{R} D(0) C(0) t .
\end{array}\right. \\
& p^{2}:\left\{\begin{array}{l}
C_{2}=\frac{1}{2} C(0)\left(2 C(0)^{2} K_{D}^{2}+3 C(0) D(0) K_{D} K_{R}+D(0)^{2} K_{R}^{2}+C(0) D(0) K_{R}^{2}\right) t^{2}, \\
D_{2}=\frac{1}{2} C(0) D(0) K_{R}\left(C(0) K_{D}+D(0) K_{R}+C(0) K_{R}\right) t^{2} .
\end{array}\right.
\end{aligned}
$$




$$
p^{3}:\left\{\begin{array}{l}
C_{3}=-\frac{1}{6} C(0)\left(6 C(0)^{3} K_{D}^{3}+12 C(0)^{2} D(0) K_{R} K_{D}^{2}+7 C(0) D(0)^{2} K_{D} K_{R}^{2}\right. \\
\left.+5 C(0)^{2} D(0) K_{D} K_{R}^{2}+D(0)^{3} K_{R}^{3}+4 C(0) D(0)^{2} K_{R}^{3}+C(0)^{2} D(0) K_{R}^{3}\right) t^{3} \\
D_{3}=-\frac{1}{6} C(0) D(0) K_{R}\left(2 C(0)^{2} K_{D}^{2}+3 C(0) D(0) K_{R} K_{D}+D(0)^{2} K_{R}^{2}\right. \\
\left.+4 C(0) D(0) K_{R}^{2}+C(0)^{2} K_{R}^{2}+3 C(0)^{2} K_{D} K_{R}\right) t^{3}
\end{array}\right.
$$

So the exact solutions of the system (1) can be entirely determined. However, in practice, all terms of the series cannot be determined, in this study we use four terms approximations:

$$
\begin{aligned}
& C(t)=\sum_{i=0}^{3} C_{i}, \\
& D(t)=\sum_{i=0}^{3} D_{i} .
\end{aligned}
$$

These approximations are presented as follows:

$$
\begin{aligned}
& C(t)=C(0)-\left(C(0)^{2} K_{D}+C(0) D(0) K_{R}\right) t+\left(C(0)^{3} K_{D}^{2}+\frac{3}{2} C(0)^{2} D(0) K_{R} K_{D}\right. \\
& \left.+\frac{1}{2} C(0) D(0)^{2} K_{R}^{2}+\frac{1}{2} D(0) C(0)^{2} K_{R}^{2}\right) t^{2}-\left(C(0)^{4} K_{D}^{3}+2 C(0)^{3} D(0) K_{R} K_{D}^{2}\right. \\
& +\frac{7}{6} C(0)^{2} D(0)^{2} K_{D} K_{R}^{2}+\frac{5}{6} D(0) C(0)^{3} K_{D} K_{R}^{2}+\frac{2}{3} C(0)^{2} D(0)^{2} K_{R}^{3} \\
& \left.+\frac{1}{6} C(0) D(0)^{3} K_{R}^{3}+\frac{1}{6} D(0) C(0)^{3} K_{R}^{3}\right) t^{3}, \\
& D(t)=D(0)-C_{(0)}{ }^{3}(0) K_{R} t+\left(\frac{1}{2} C(0)^{2} D(0) K_{R}^{2}+\frac{1}{2} C(0)^{2} D(0) K_{R} K_{D}\right. \\
& \left.+\frac{1}{2} C(0) D(0)^{2} K_{R}^{2}\right) t^{2}-\left(\frac{1}{3} C(0)^{3} D(0) K_{D}^{2} K_{R}+\frac{1}{2} C(0)^{2} D(0)^{2} K_{D} K_{R}^{2}\right. \\
& +\frac{1}{6} C(0) D(0)^{3} K_{R}^{3}+\frac{2}{3} D(0)^{2} C(0)^{2} K_{R}^{2}+\frac{1}{2} C(0)^{3} D(0) K_{R}^{2} K_{D} \\
& \left.+\frac{1}{6} D(0) C(0)^{3} K_{R}^{3}\right) t^{3} .
\end{aligned}
$$


Table 1 The model parameters and constants

\begin{tabular}{|l|c|}
\hline Parameter & Value \\
\hline$E, \mathrm{kcal} / \mathrm{mol}$ & $8.0 \times 10^{4}$ \\
\hline$R, \mathrm{~kJ} / \mathrm{K} \mathrm{mol}$ & 8.314 \\
\hline$A D, l / \mathrm{min}$ & $10^{9}$ \\
\hline$A R, l / \mathrm{min}$ & $0.5 \times 10^{9}$ \\
\hline$C(0), \mathrm{mg} / \mathrm{l}$ & 2 \\
\hline$D(0), \mathrm{mg} / l$ & $10^{-5}$ \\
\hline$O H$ & $0.8 \times 10^{-9}$ \\
\hline
\end{tabular}

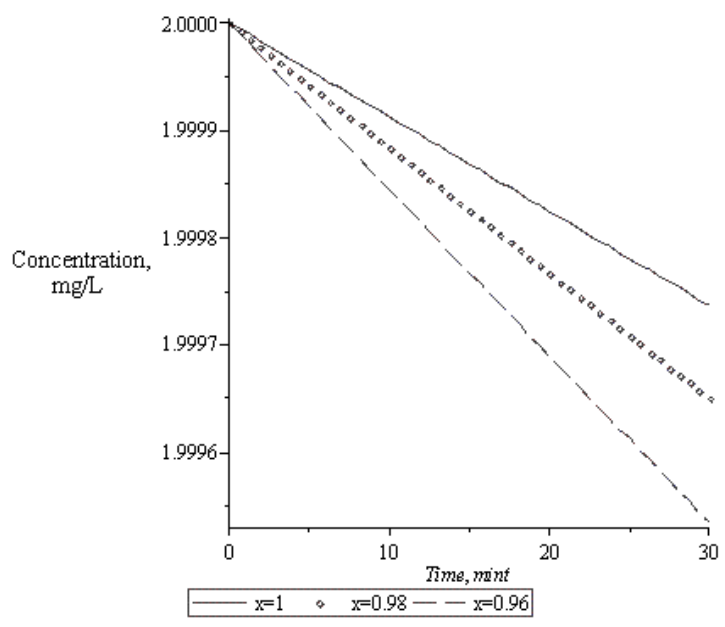

Fig. 1. Plots of the second-order reaction for different values of $x$ versus time 


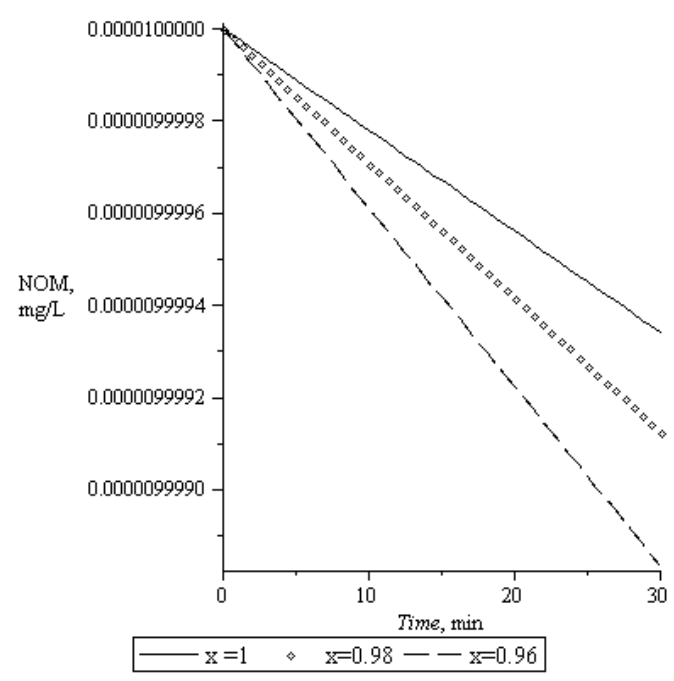

Fig.2. Plots of the second-order reaction NOM for different value of $\mathrm{x}$ versus time

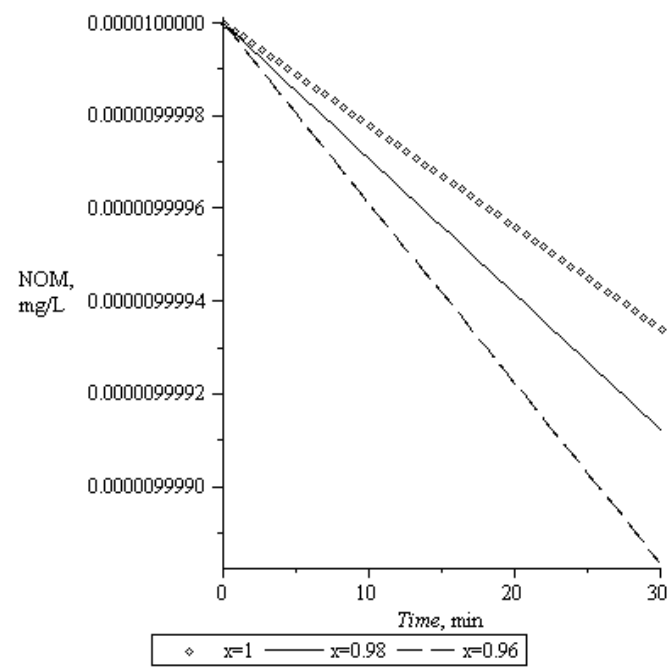

Fig. 3. Plots of first-order reaction NOM versus time 


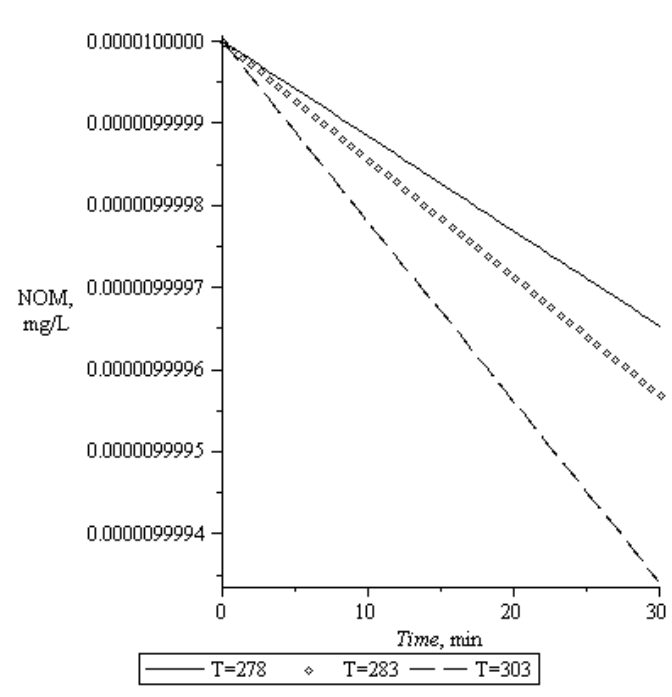

Fig.4. Plots of the second-order reaction NOM for different temperatures versus time

\section{DISCUSSION}

For numerical study let us start with stating the relationship of $K_{D}$ and $K_{R}$ with other parameters as

$$
\begin{aligned}
& K_{D}=A_{D}\left[O H^{-}\right]^{x} \exp \left(-\frac{E}{R T}\right), \\
& K_{R}=A_{R}\left[O H^{-}\right]^{x} \exp \left(-\frac{E}{R T}\right) .
\end{aligned}
$$

where $A_{D}$ and $A_{R}$, frequency factors for ozone decomposition reactions (1/ $\min ) ;\left[\mathrm{OH}^{-}\right]$, concentration of hydroxide ion (mol / 1); X, reaction order; $E$, activation energy ( $\mathrm{kcal} / \mathrm{mol}) ; R$, gas constant (kJ/K mol); $T$, temperature (K). In this study the values in Table 1 are considered.

Fig. 1 presents the behavior of ozone concentration and NOM; the values of the parameters from Table 1 are substituted in the solutions. 
Fig. 1 shows the changes in residual ozone with time. The results suggest that ozone decomposes at a faster rate as with increases in the reaction order of the hydroxyl ion.

Fig. 2 shows the effect of temperature on the ozone decomposition. The residual ozone concentration decreases at a faster rate, as temperature of the marine water was increase from $5^{\circ} \mathrm{C}$ to $15^{\circ} \mathrm{C}$.

Fig. 3 shows the effect of hydroxyl ion reaction order on the degradation of the natural organic matter. The NOM degrades at a faster rate as the reaction order of the hydroxyl ion decreases.

Fig. 4 shows the effect of temperature on the degradation of the natural organic matter. The NOM degrades at a faster rate as the temperature increases.

A nonlinear system of differential equations, governing on the ozone decomposition, has been solved by HPM, which seems to be very easy and accurate to employ with reliable results.

For computations and plots we used Maple 11.

\section{REFERENCES}

[1] S.T.Summer felt, J.A. Hankins, A.L. Weber, M.D. Durant, Ozone ion of a re-circulating rainbow trout culture system. II. Effects on micro screen filtration and water quality, Aquaculture 158 (1997) 57-67.

[2] G.L. Lucchetti, G.A. Gray, Water reuses systems, a review of principal components, Prog. Fish Culturist. 50 (1988) 1-6.

[3] J. Biazar, M. Tango, R. Islam, Ozone decomposition of the second order in aqueous solutions, Applied Mathematics and Computation 177 (2006) 220-225.

[4] J. Biazar, H. Ghazvini Exact solutions for nonlinear Schrödinger equations by He's homotopy perturbation method Physics Letters A 366 (2007), 79-84.

[5] SJ. Liao, An approximate solution technique not depending on small parameter: a special example. In J Nonlinear Mech 1995:30(3):371-80.

[6] SJ. Liao, Boundary element method for general nonlinear differential operators. Eng Anal Boundary Element 1997; 20(2):91-9.

[7] J.H. He, The homotopy perturbation method for nonlinear oscillators with discontinuities, Applied Mathematics and Computation 151 (2004) 287-292.

[8] J. Biazar, M. Eslami, H. Ghazvini, Homotopy perturbation method for systems of partial differential equations, International Journal of Nonlinear Science and Numerical Simulation 8 (3) (2007) 411 416.

[9] J.H. He, Comparison of homotopy perturbation method and homotopy analysis method, Applied Mathematics and Computation 156 (2004) 527-539.

[10] J.H. He, Asymptology by homotopy perturbation method, Applied Mathematics and Computation 156 (3) (2004) 591-596. 
[11] J.H. He, Homotopy perturbation method for bifurcation of nonlinear problems, International Journal of Nonlinear Science Numerical Simulation 6 (2) (2005) 207-208.

[12] J.H. He, Homotopy perturbation method for solving boundary value problems, Physics Letters A 350 (1-2) (2006) 87-88.

[13] J.H. He, Homotopy perturbation technique, Computer methods in applied mechanics and engineering 178 (1999) 257-262.

\title{
Z. Ayati
}

Department of Engineering sciences,

Faculty of Technology and Engineering East of Guilan, University of Guilan, P.C. 44891-63157, Rudsar-Vajargah, Iran

E-mail address: Ayati.zainab@gmail.com \& zainab.ayati@guilan.ac.ir

\section{J. Biazar}

Department of Applied Mathematics

Faculty of Mathematical Science, University of Guilan, , P.O. Box 41635-19141, P.C. 4193833697, Rasht, Iran

\author{
M. Partovi \\ Department of Applied Mathematics \\ Faculty of Mathematical Science, University of Guilan , \\ P.O. Box 41635-19141, P.C. 4193833697, Rasht, Iran
}

